\title{
Cultural differences in statistical approximations to English
}

NEVILLE MORAY

UNIVERSITY OF SHEFFIELD, ENGLAND

The relatively small amount of material which has been prepared for use in experiments on statistical approximations to English means that there may be marked differences in its statistical properties from one sample to another. The present paper investigates the relation between the source of the sample and the amount of repetition which occurs at any level of approximation, showing marked disparities between American and English samples.

Of the four sets of statistical approximations known to the writer, two come from American sources (Miller \& Selfridge, 1954; Deese, 1959) and two from English sources (Taylor \& Moray, 1960; Morton, 1965). Treisman (1964) has recently published an estimate of the information content of this material based upon a guessing technique and Harrison, Moray, and Treisman have checked the guesses for their closeness to asymptomatic values for the information content. Morton has pointed out that if punctuation is allowed a very different kind of estimate may be obtained, and since the absolute length of the samples is rather short, their use as a measuring device when investigating speech performance is clearly a matter requiring care if there are marked differences in their properties.

In the present investigation an attempt was made to measure the amount of repetition in the different orders of approximation for the two American samples and one British one.

Method

Two measures have been used to estimate repetition. The first is the well known Type/Token ratio. The second is the Statistic $K$ of Yule, originally invented to investigate the provenance of literary documents (Yule, 1944). It has the advantage over the TTR of using the square of the frequency of occurrence in its calculation, thus rendering it much more sensitive to slight increases in the repetition frequency of words.

The TTR and $\mathrm{K}$ were calculated for each sample of "prose." In the case of the samples due to Deese, the

Table 1. Type Token Ratios and Order of Approximation

\begin{tabular}{lccccccccc} 
& 2nd & 3rd & 4th & 5th & 6th & 7th & 8th & 12th & 16th \\
\hline $\begin{array}{l}\text { Deese } \\
\text { Miller and }\end{array}$ & 0.66 & 0.82 & 0.80 & 0.81 & 0.84 & - & 0.34 & - & - \\
$\quad$ Selfridge & 0.65 & 0.71 & 0.74 & 0.74 & $-0.71-$ & - & - \\
$\begin{array}{l}\text { Taylor and } \\
\text { Moray }\end{array}$ & $0.81-0.77-0.78-0.790 .750 .77$ \\
\hline
\end{tabular}

value given is the average obtained by pooling the several examples of each order which he obtained.

The results are shown in Tables I and II.

\section{Discassion}

It will be noticed that there is rather little difference between the samples as measured by the TTR. On the other hand, Yule's $\mathrm{K}$ shows very marked differences both between different orders of approximation from different sources. The slope of the function relating $K$ to order of approximation is positive for the British sample, and negative for the American samples. In fact the two cultures provide curves which are almost orthogonal to one another.

A high value of $K$ means that words are being frequently repeated within the passage, i.e., that a functionally small vocabulary is being used to generate it. Deese (personal communication) suggested that a high $K$ is to be expected at low orders of approximation, since where there are no contextual constraints, words will be chosen by the Ss to construct the approximations in a way which matches the frequency of usage of the words in the language, and that the amount of repetition should fall when there is a definite topic to discuss. The present writer predicted that words would be more frequently repeated where the context was sufficiently well specified to reduce the set from which the next word could be chosen, and be less repeated where they could be chosen at random. Both predictions have been verified.

It seems likely that there is a cultural determinant which is reflected in what we may call the "functional vocabulary size" on which Ss draw when asked for the next word in a sentence. We might expect that the two curves found represent two of a family of such curves, reflecting the way in which Ss approach the task of retrieving words from the memory store and the criteria they set themselves when faced with such a task. A similar difference is probably reflected in the prediction by Deese and the writer as to which "word game" our respective students would play.

Table 2. Yule's "K" and Order of Approximation

\begin{tabular}{|c|c|c|c|c|c|c|c|c|c|}
\hline & \multicolumn{9}{|c|}{ Order } \\
\hline & 2nd & 3rd & 4th & 5th & 6th & 7 th & 8 th & 12 th & 16 th \\
\hline Deese & 3.39 & 114 & 127 & 128 & 110 & - & 89 & - & - \\
\hline Miller and & & & & & & & & & \\
\hline Selfridge & 156 & 146 & 106 & 92 & - & 94 & - & - & - \\
\hline Moray & 41 & - & 40 & - & 62 & - & 64 & 96 & 110 \\
\hline
\end{tabular}




\section{neforences}

Deese, J. Personal communication, 1959.

Miller, G. A., \& Selfridge Verbal context and the recall of meaningful material. Amer. J. Psychol., 1950, 63, 167-185.

Morton, J. Personal communication.
Taylor, A., \& Moray, N. Statistical approximations to English and French. Lang. Speech, 1960, 3, 7-10.

Treisman, A. Verbal cues, language and meaning in selective attention. Amer. J. Psychol., 1964, 77, 206-19.

Yule, $\mathbf{0}$. The statistical study of literary vocabulary. Cambridge: Cambridge University Press, 1944. 